\title{
Daptomycin: a comparison of two intravenous formulations
}

This article was published in the following Dove Press journal:

Drug Design, Development and Therapy

\section{Celeste Frankenfeld \\ Sachin Mittal \\ Yanira Melendez \\ Luke Mendez-Vigo \\ Kenneth C Lamp \\ Kaitlin N Keller \\ Shellie R Bertolami}

Merck \& Co., Inc., Kenilworth, NJ, USA
Correspondence: Celeste Frankenfeld Global CMC Regulatory Affairs, Merck Research Laboratories, I 26 E. Lincoln Avenue, PO Box 2000, Rahway, NJ 07065, USA

$\mathrm{Tel}+\mathrm{I} 7325942504$

Fax + I 7325941780

Email celeste.frankenfeld@merck.com

\begin{abstract}
Daptomycin is a cyclic lipopeptide antibacterial agent with potent bactericidal activity against a broad range of Gram-positive organisms. In 2003, daptomycin for injection received approval from the US Food and Drug Administration (FDA) for the treatment of patients with complicated skin and skin structure infections (cSSSIs); in 2006, it was approved for the treatment of patients with Staphylococcus aureus bacteremia, including those with right-sided infective endocarditis caused by methicillin-susceptible and methicillin-resistant isolates. In 2016, the FDA approved a new formulation of daptomycin for injection (daptomycin RF) for the same indications. The efficacy and safety of daptomycin for injection have been established in pivotal clinical trials, and the findings of nonclinical studies indicate that both formulations of daptomycin for injection are equivalent. Herein we refer to the new daptomycin formulation as daptomycin RF to distinguish it from the original formulation. Daptomycin RF provides clinicians and clinical pharmacists with a product that offers improved stability and more rapid, in-vial reconstitution with either sterile or bacteriostatic water for injection, while maintaining the same antibacterial coverage. Here we discuss the rationale for and the potential value of daptomycin RF, and briefly review the similarities and differences between the original formulation of daptomycin and daptomycin RF.
\end{abstract}

Keywords: MRSA, drug stability, Gram-positive bacterial infections, bacteremia, antibiotics, formulation

\section{Plain language summary}

Daptomycin is an intravenously administered antibiotic. Daptomycin, first approved by the US Food and Drug Administration (FDA) in 2003, is effective against a broad range of bacterial infections, including those caused by methicillin-resistant Staphylococcus aureus (MRSA). Daptomycin has been used to treat patients with bacterial infections of the skin and underlying tissues as well as infections that have entered the bloodstream. Daptomycin is provided by the manufacturer as a powder that requires mixing with a liquid before injection. In 2016, the FDA approved a new formulation of daptomycin (daptomycin RF) that shortens the time required to dissolve the powder into a solution and improves the use period of the prepared solution, while maintaining the antibiotic's strength to fight the same types of bacterial infections. This article highlights the differences in the new formulation, compared with the original. Key differences of the new formulation include: 1) The powder can be stored at room temperature. 2) It is quicker and easier to prepare the injection/infusion solution from the powder. 3) The powder should be dissolved in sterile or bacteriostatic water instead of saline solution. 4) It has a longer use period of injection/infusion solution - both at room temperature or with refrigeration. 


\section{Introduction}

Daptomycin is a cyclic lipopeptide antibacterial agent with potent bactericidal activity against a broad range of Grampositive organisms. ${ }^{1,2}$ On the basis of 2 pivotal randomized, controlled, Phase III clinical trials, the US Food and Drug Administration (FDA) approved daptomycin for injection (Cubicin ${ }^{\circledR}$; Merck \& Co., Inc., Kenilworth, NJ, USA) for the treatment of complicated skin and skin structure infections (cSSSIs) caused by certain Gram-positive bacteria in 2003 and for the treatment of Staphylococcus aureus bloodstream infections (bacteremia), including right-sided infective endocarditis, caused by methicillin-susceptible and methicillin-resistant isolates in $2006{ }^{4-6}$

Stability, extended use period, and reduced reconstitution time are the important attributes for a lyophilized antibacterial agent intended for intravenous (IV) administration. A new formulation of daptomycin for injection (daptomycin $\mathrm{RF}$ ) has been developed that provides improved stability (eg, room temperature storage) and faster reconstitution time, while maintaining the same antibacterial coverage as the original formulation. ${ }^{4,7}$ In 2016, the FDA approved daptomycin RF (Cubicin RF; Merck \& Co., Inc.) for the same indications as those of the original daptomycin formulation. ${ }^{3,7}$ Both formulations of daptomycin also received approval in 2017 for the treatment of cSSSIs and S. aureus bloodstream infections (bacteremia) in pediatric patients (aged 1-17 years).

Here we review the rationale for and the potential value of daptomycin RF, highlighting its similarities to and differences from the original approved formulation of daptomycin, and improvements that have been made to its product-handling characteristics.

\section{Similarities and differences between daptomycin and daptomycin RF Formulation, stability, and reconstitution}

Table 1 summarizes the major differences between daptomycin and daptomycin RF.,7 Daptomycin RF has the same indication, dosage form (lyophilized in-vial presentations and $500 \mathrm{mg}$ strength in the USA), and administration recommendations for adults ( 2 minutes for IV injection; 30 minutes for IV infusion) as the original formulation of daptomycin. However, pediatric patients should only receive daptomycin by IV infusion for 30 minutes, regardless of formulation., ${ }^{4,7}$ The active ingredient of both daptomycin RF and daptomycin is the same, but the formulations are not identical.

Two key differences between the formulations are the addition of sucrose as an inactive ingredient and an increased target $\mathrm{pH}$ (6.8 vs 4.7) in daptomycin RF that is achieved during manufacturing through titration with sodium hydroxide. ${ }^{4,7}$ The addition of sucrose enhances the shelf stability of daptomycin RF, permitting the storage of the lyophilized powder vial at controlled room temperature $\left(20^{\circ} \mathrm{C}-25^{\circ} \mathrm{C}\right)$ rather than under refrigeration $\left(5^{\circ} \mathrm{C} \pm 3^{\circ} \mathrm{C}\right)$; refrigerated storage is required for the original formulation of daptomycin. ${ }^{4,7}$

The increased $\mathrm{pH}$ of daptomycin $\mathrm{RF}$ reduces reconstitution time compared with daptomycin ( $\sim 2$ vs $\sim 15$ minutes, respectively). Preparation of daptomycin requires gentle rotation after the addition of diluent, followed by a 10-minute rest period and a further swirling to ensure thorough reconstitution; vigorous agitation is discouraged to minimize foaming. Preparation of daptomycin RF is far less complex and only requires gentle rotation or swirling for a few minutes to completely reconstitute the lyophilized powder., ${ }^{4,7}$

Furthermore, the diluent for vial reconstitution of daptomycin RF has been changed from $0.9 \%$ sodium chloride to sterile or bacteriostatic water for injection due to the addition of sucrose. ${ }^{4,7}$ This change is particularly useful in the context of historic saline shortages, where daptomycin RF can also be administered through IV injection over a 2-minute period using sterile or bacteriostatic water for injection if $0.9 \%$ sodium chloride is not available. ${ }^{7}$ Use of a saline diluent for reconstitution of daptomycin RF may result in a hypertonic solution, increasing the risk for injection site irritation if administered by 2 -minute IV injection. However, a $0.9 \%$ sodium chloride diluent can still be used with daptomycin RF when the reconstituted product is further diluted in an IV bag. ${ }^{7}$ In the case that sterile or bacteriostatic water for injection is not available, the original daptomycin formulation may be used, as it requires reconstitution in $0.9 \%$ sodium chloride alone. ${ }^{4}$

The reformulation of daptomycin has yielded a more stable product in daptomycin RF. Daptomycin RF has a longer in-use shelf period although times vary based on dosage form (vial, IV bag, or syringe) and diluent used. ${ }^{4,7}$ For example, daptomycin $\mathrm{RF}$ reconstituted in the vial with bacteriostatic water or sterile water and subsequently refrigerated has an in-use storage period of 3 days, and daptomycin RF reconstituted in the vial with sterile water and stored at room temperature has an in-use storage period of 1 day. ${ }^{7}$ In contrast, the original formulation must be refrigerated prior to reconstitution, and the diluted solution has an in-use storage period of 48 hours with refrigeration. ${ }^{4}$ In order to reduce confusion if both products are available in the same location, the product packaging features a blue cap for daptomycin and a purple cap for daptomycin RF. 
Table I Major differences between daptomycin and daptomycin RF

\begin{tabular}{|c|c|c|c|}
\hline Characteristic & Daptomycin for injection for IV use & \multicolumn{2}{|c|}{ Daptomycin RF for injection for IV use } \\
\hline Vial storage & - Refrigerated $\left(2^{\circ} \mathrm{C}-8^{\circ} \mathrm{C}\left[36^{\circ} \mathrm{F}-46^{\circ} \mathrm{F}\right]\right)$ & \multicolumn{2}{|c|}{$\begin{array}{l}\text { - Controlled room temperature }\left(20^{\circ} \mathrm{C}-25^{\circ} \mathrm{C}\left[68^{\circ} \mathrm{F}-77^{\circ} \mathrm{F}\right] \text {; }\right. \\
\left.\text { excursions permitted to } 15^{\circ} \mathrm{C}-30^{\circ} \mathrm{C}\left[59^{\circ} \mathrm{F}-86^{\circ} \mathrm{F}\right]\right)\end{array}$} \\
\hline Diluent for vial reconstitution & - $0.9 \%$ sodium chloride & \multicolumn{2}{|c|}{ - Sterile water or bacteriostatic water for injection } \\
\hline Compatible IV solutions & $\begin{array}{l}\text { Daptomycin is compatible with } 0.9 \% \text { sodium } \\
\text { chloride injection and lactated Ringer's injection }\end{array}$ & \multicolumn{2}{|c|}{$\begin{array}{l}\text { Reconstituted daptomycin RF is compatible with sterile } \\
\text { water for injection, bacteriostatic water for injection, and } \\
0.9 \% \text { sodium chloride injection }\end{array}$} \\
\hline $\begin{array}{l}\text { Reconstitution of vial } \\
\text { in preparation for } \\
\text { administration }\end{array}$ & $\begin{array}{l}\text { - To minimize foaming, AVOID vigorous agitation } \\
\text { or shaking of the vial during or after } \\
\text { reconstitution } \\
\text { - Ensure that all the daptomycin powder is } \\
\text { wetted by gently rotating the vial } \\
\text { - Allow the wetted product to stand undisturbed } \\
\text { for } 10 \text { minutes before gently rotating or } \\
\text { swirling the vial contents for a few minutes to } \\
\text { reconstitute }\end{array}$ & \multicolumn{2}{|c|}{$\begin{array}{l}\text { - Upon wetting the daptomycin RF powder with the } \\
\text { diluent, the vial contents can be rotated and swirled } \\
\text { immediately for a few minutes to reconstitute }\end{array}$} \\
\hline $\begin{array}{l}\text { Approximate osmolality of } \\
50 \mathrm{mg} / \mathrm{mL}\end{array}$ & $\begin{array}{l}\text { - } 364 \mathrm{mOsm} / \mathrm{kg} \text { when reconstituted in } 0.9 \% \\
\text { sodium chloride injection }\end{array}$ & \multicolumn{2}{|c|}{ - $323 \mathrm{mOsm} / \mathrm{kg}$ when reconstituted in water for injection } \\
\hline \multicolumn{4}{|c|}{ In-use storage period (once reconstituted) } \\
\hline Vial & Sodium chloride for injection & Sterile water for injection & $\begin{array}{l}\text { Bacteriostatic water for } \\
\text { injection }\end{array}$ \\
\hline Room temperature ${ }^{b}$ & - 12 hours & - I day & - 2 days \\
\hline Refrigerated $^{c}$ & - 48 hours & - 3 days & - 3 days $^{\mathrm{d}}$ \\
\hline IV bag preparation & $\begin{array}{l}\text { Reconstitution: } 0.9 \% \text { sodium chloride for injection } \\
\text { for immediate dilution with } 0.9 \% \text { sodium chloride } \\
\text { injection }\end{array}$ & $\begin{array}{l}\text { Reconstitution: sterile water } \\
\text { for injection for immediate } \\
\text { dilution with } 0.9 \% \text { sodium } \\
\text { chloride injection }\end{array}$ & $\begin{array}{l}\text { Reconstitution: } \\
\text { bacteriostatic water for } \\
\text { injection for immediate } \\
\text { dilution with } 0.9 \% \text { sodium } \\
\text { chloride injection }\end{array}$ \\
\hline Room temperature ${ }^{b}$ & - 12 hours & - 19 hours & - 2 days \\
\hline Refrigerated $^{c}$ & - 48 hours & - 3 days & - 5 days \\
\hline Syringe & $\begin{array}{l}\text { No in-use storage period specified in prescribing } \\
\text { information }\end{array}$ & Sterile water for injection & $\begin{array}{l}\text { Bacteriostatic water for } \\
\text { injection }\end{array}$ \\
\hline Room temperature ${ }^{b}$ & - N/A & - I day & - 2 days \\
\hline Refrigerated $^{c}$ & - N/A & - 3 days & - 5 days \\
\hline
\end{tabular}

Notes: Data from package inserts of Cubicin ${ }^{2}$ (daptomycin for injection) ${ }^{4}$ and Cubicin $\circledast$ RF (daptomycin for injection). ${ }^{7}$ Osmolality refers to the number of solute particles per kilogram of water; osmolarity refers to the number of solute particles per liter of water. Osmolarity is considered roughly equivalent to osmolality (ie, I $\mathrm{L}$ is approximately equal to I kg) because normal saline is a dilute aqueous solution with a specific gravity of 1.0003 . ${ }^{\circ}$ Room temperature $=20^{\circ} \mathrm{C}-25^{\circ} \mathrm{C}\left(68^{\circ} \mathrm{F}-77^{\circ} \mathrm{F}\right)$. ${ }^{\mathrm{C}}$ Refrigerated temperature $=2^{\circ} \mathrm{C}-8^{\circ} \mathrm{C}\left(36^{\circ} \mathrm{F}-46^{\circ} \mathrm{F}\right)$. ${ }^{d}$ Storage period is determined by sterility rather than stability of reconstituted product. Daptomycin RF is a new formulation of daptomycin for injection.

Abbreviations: IV, intravenous; N/A, not applicable.

\section{Compatibility of daptomycin}

Daptomycin is compatible with $0.9 \%$ sodium chloride solution and lactated Ringer's solution. ${ }^{4}$ Daptomycin RF is chemically compatible with sterile water for injection, bacteriostatic water for injection, $0.9 \%$ sodium chloride solution, or lactated Ringer's solution; however, initial reconstitution should only occur with sterile or bacteriostatic water for injection due to osmolality constraints and to prevent injection site irritation. ${ }^{7}$ Both daptomycin and daptomycin $\mathrm{RF}$ are incompatible with dextrose-containing diluents and should not be used in conjunction with ReadyMED (Reliant
Medical Group, Worcester, MA, USA) elastomeric infusion pumps (impurity identified). ${ }^{4,7}$

Physical and chemical potency analyses demonstrated that daptomycin was stable and compatible when admixed with commonly administered IV medications, including aztreonam, ceftazidime, ceftriaxone, dopamine, gentamicin, fluconazole, heparin, levofloxacin, and lidocaine. ${ }^{8}$ However, unpublished studies conducted by Merck demonstrated that both daptomycin and daptomycin RF reconstituted solutions were incompatible when admixed with reconstituted vancomycin (data on file, Merck \& Co., Inc.). 


\section{Establishing the equivalence of daptomycin RF and daptomycin}

Evidence of clinical equivalence between the daptomycin and daptomycin RF formulations was obtained through comparative evaluation of data generated from a series of in vitro studies and through evaluation of the available literature; these findings were presented to the FDA before it granted approval for daptomycin RF. ${ }^{3}$ The justifications supporting the clinical equivalence of daptomycin RF and daptomycin can be articulated as follows:

1. Per the Code of Federal Regulations 21CFR320.22(b) (1), the in vivo bioequivalence of a drug product may be considered self-evident if it is a parenteral solution intended solely for administration by injection and contains the same active and inactive ingredients in the same concentrations as the approved drug product. ${ }^{9}$ Daptomycin RF is a parenteral solution intended solely for IV infusion and, following reconstitution, contains the same daptomycin drug concentration as the current marketed product; therefore, the in vivo bioequivalence is self-evident for this formulation. However, the impact of the additional formulation adjustments must also be taken into consideration (eg, addition of sucrose). To that end, an additional literature research and additional studies were conducted as described in justifications 2-6. ${ }^{3}$

2. The potential for differences in pharmacokinetic (PK) parameters between the 2 formulations was also evaluated in a crossover PK animal study (beagle dogs). ${ }^{3}$ This model has been used effectively to translate the exposureresponse relationship for skeletal muscle safety to the clinical situation. A single IV bolus injection of $15 \mathrm{mg} / \mathrm{kg}$ diluted in either distilled water (daptomycin RF) or saline (daptomycin) was delivered to 6 male dogs on Day 1 followed by another single IV bolus injection of the same dilutions on Day 8. Plasma concentrations were assessed from samples taken at time points from 2 minutes to 24 hours post injection. There were no significant differences between daptomycin RF and daptomycin in the plasma concentrations, area under the concentration-time curve from time zero extrapolated to infinity $\left(\mathrm{AUC}_{0-\infty}\right.$; 768 vs $807 \mu \mathrm{g} \times \mathrm{h} / \mathrm{mL}$ ), or maximum plasma concentration $\left(C_{\max } ; 258\right.$ vs $\left.257 \mu \mathrm{g} / \mathrm{mL}\right)$. Likewise, no differences were detected between daptomycin RF and daptomycin in the mean values for plasma clearance $(0.33$ vs $0.32 \mathrm{~mL} / \mathrm{min} / \mathrm{kg}$ ), volume of distribution at steady state ( $80 \mathrm{vs} 90 \mathrm{~mL} / \mathrm{kg}$ ), and terminal elimination half-life (2.8 vs 3.4 hours). The findings of this study indicated that the PK of daptomycin RF was unchanged compared with that of daptomycin, demonstrating the comparability of the 2 formulations. ${ }^{3}$

3. A 2-week toxicity and toxicokinetic study was conducted in rats to identify potential changes to the nonclinical safety profile of sucrose-based daptomycin RF. The highest tolerated dose of $100 \mathrm{mg} / \mathrm{kg}$ was administered to 2 male and 2 female rats. At this dose, no mortality or clinical signs were detected for up to 24 hours after dosing so $100 \mathrm{mg} / \mathrm{kg}$ was selected as the high dose for a 2 -week study. Ten rats per group were dosed once daily for 14 days with either daptomycin RF or daptomycin at concentrations of $0,25,50$, or $100 \mathrm{mg} / \mathrm{kg} /$ day. For both groups at all doses, there were no deaths, clinical signs observed, or changes in body weight or food consumption. Hematology/coagulation, clinical chemistry, and urine analysis findings were not significant or toxicologically relevant. Toxicokinetic parameters including area under the concentration-time curve from time 0 to 24 hours $\left(\mathrm{AUC}_{0-24}\right)$, and $C_{\text {max }}$ were similar between daptomycin RF and daptomycin. Further, a comparison of hemolytic potential of the 2 formulations showed no difference in any tested concentration $(50-200 \mu \mathrm{g} / \mathrm{mL})$. Overall, findings for daptomycin RF were comparable to previous results with the original formulation. All changes of toxicologic significance were previously noted in rats and were not significantly impacted by daptomycin RF.,4

4. Per the Code of Federal Regulations 21CFR320.22(d) (4), bioequivalence of a drug product may be measured in vitro in lieu of clinical data if it is a reformulated product that is identical except for a different color, flavor, or preservative that could not affect its bioavailability. ${ }^{9}$ Physicochemical compatibility data demonstrated that there were no interactions between daptomycin and the added excipients (ie, sucrose) that could affect the bioavailability of the product. Furthermore, in vitro studies demonstrated no difference in protein binding or microbiologic potency between daptomycin RF and daptomycin. Specifically, plasma protein binding was evaluated via equilibrium dialysis, and at 10 and $100 \mu \mathrm{g} / \mathrm{mL}$ of daptomycin, both formulations showed nearly identical percentages of protein binding, ranging between $89.3 \%$ and $93.2 \%$ at the lower concentration and $94.4 \%-95.3 \%$ at the higher concentration. ${ }^{3}$ Taken together, these data indicate that free drug concentration and microbiologic activity are similar between the two formulations; hence, efficacy is also expected to be similar.

5. The sole additional inactive ingredient in daptomycin RF is sucrose, which is generally recognized as safe and is 
commonly used in many FDA-approved parenteral drug products. Thorough evaluation of the available literature did not reveal any evidence that the amount of sucrose in daptomycin RF would impact the distribution or elimination of daptomycin. In addition, the sucrose level on reconstitution of daptomycin RF in a vial is $7.5 \%$ weight per volume (wt/vol), which is well below the maximum acceptable level of sucrose in a drug product for administration by IV injection (19.5\% wt/vol), as indicated by the FDA's Inactive Ingredient Database (IID). ${ }^{10}$ The IID provides information (eg, route, dosage form, and maximum potency) on inactive ingredients in FDA-approved drug products; an inactive ingredient that is approved and included in the IID at a certain dosage form and potency may be considered safe if it is used in a similar manner for a similar type of drug product (eg, IV administration by injection). ${ }^{10}$ Furthermore, the amount of sucrose that would be administered to an average $80 \mathrm{~kg}$ patient taking daptomycin RF $6 \mathrm{mg} / \mathrm{kg}$ is $4.8 \mathrm{~g} /$ week, which is well below the threshold for renal injury of $1 \mathrm{~g} / \mathrm{kg}$ per week in an $80 \mathrm{~kg}$ patient with renal insufficiency. ${ }^{11}$ In addition, the amount of sucrose on reconstitution of daptomycin $\mathrm{RF}(0.75 \mathrm{~g}$ in $10 \mathrm{~mL}$ sterile water for injection) is not anticipated to have a clinically meaningful impact on blood glucose in patients. Earlier studies have found no impact on blood glucose levels after oral intake of sucrose at doses of 15-25 $\mathrm{g}$ in patients with well-controlled or poorly controlled diabetes. ${ }^{12,13}$

6. The $\mathrm{pH}$ and osmolality of daptomycin RF were evaluated for potential issues related to compatibility with blood and local tolerability at the injection site. ${ }^{3}$ The higher $\mathrm{pH}$ of daptomycin RF suggests greater compatibility with physiologic pH, with no impact on bioavailability. Although the osmolality of daptomycin RF is increased, it remains within a compatible range for infusion when daptomycin is reconstituted correctly and is not considered to have a clinically meaningful impact on the tolerability of the new IV formulation. ${ }^{3}$

7. In vitro studies, dilution compatibility studies, and stability studies comparing daptomycin RF and daptomycin were conducted. The findings of these studies indicated that the dilution compatibility of reconstituted daptomycin RF with sterile or bacteriostatic water for injection, $0.9 \%$ sodium chloride, or lactated Ringer's solution was equivalent or better than that of daptomycin.

8. Product stability and degradation profile remained comparable or better with daptomycin RF vs daptomycin.

Collectively, these data demonstrated the comparability of daptomycin RF with daptomycin, and in 2016, the FDA approved daptomycin RF for the same indications and for use in the same manner as daptomycin. . $^{3,4}$,

\section{Conclusion}

Daptomycin RF is an alternative option for the treatment of patients with cSSSIs and $S$. aureus bloodstream infections; this new formulation is available in the USA and Canada. The efficacy and safety of daptomycin for injection have been established in pivotal clinical trials, and the findings of nonclinical studies indicate that both formulations of daptomycin for injection are equivalent. In the outpatient setting, the improved time to reach complete solution during reconstitution requires less nursing attention for the overall process, and a $\mathrm{pH}$ closer to physiologic values will continue to support the usefulness of daptomycin RF in this setting as either an IV injection or infusion. In addition, room temperature storage conditions and shorter reconstitution times may make daptomycin RF more easily used in an outpatient setting such as a hemodialysis clinic (note: when possible, daptomycin RF should be administered following the completion of hemodialysis). ${ }^{7}$

In conclusion, this new formulation provides patients and health care providers (eg, physicians and clinical pharmacists) with a therapeutic option that offers greater stability and requires less time for reconstitution.

\section{Acknowledgments}

The authors thank Sonia Atzingen of Merck \& Co., Inc., Kenilworth, NJ, USA, for her contributions to this research. Medical writing and editorial assistance were provided by Maxwell Chang, BSc(Hons), and Timothy A. Becker, PharmD, ApotheCom, Yardley, PA, USA. This assistance was funded by Merck \& Co., Inc.

\section{Author contributions}

All authors contributed toward data analysis, drafting, and critically revising the paper; gave final approval of the version to be published; and agreed to be accountable for all aspects of the work.

\section{Disclosure}

All authors are employees of Merck \& Co., Inc., Kenilworth, NJ, USA. The authors report no other conflicts of interest in this work.

\section{References}

1. Sauermann R, Rothenburger M, Graninger W, Joukhadar C. Daptomycin: a review 4 years after first approval. Pharmacology. 2008;81(2): 79-91. 
2. Gonzalez-Ruiz A, Seaton RA, Hamed K. Daptomycin: an evidence-based review of its role in the treatment of Gram-positive infections. Infect Drug Resist. 2016;9:47-58.

3. US Food and Drug Administration (Center for Drug Evaluation and Research). Approval Package for NDA 21-572/S-052 (Cubicin RF). 2016.

4. Cubicin ${ }^{\circledR}$ (daptomycin for injection) for intravenous use [package insert]. Whitehouse Station, NJ: Merck \& Co., Inc.; 2017.

5. Arbeit RD, Maki D, Tally FP, Campanaro E, Eisenstein BI; Daptomycin 98-01 and 99-01 Investigators. The safety and efficacy of daptomycin for the treatment of complicated skin and skin-structure infections. Clin Infect Dis. 2004;38(12):1673-1681.

6. Fowler VG Jr, Boucher HW, Corey GR, et al; S. aureus Endocarditis and Bacteremia Study Group. Daptomycin versus standard therapy for bacteremia and endocarditis caused by Staphylococcus aureus. $N$ Engl J Med. 2006;355(7):653-665.

7. Cubicin ${ }^{\circledR}$ RF (daptomycin for injection) for intravenous use [package insert]. Whitehouse Station, NJ: Merck \& Co., Inc.; 2017.

8. Lai JJ, Brodeur SK. Physical and chemical compatibility of daptomycin with nine medications. Ann Pharmacother. 2004;38(10):1612-1616.
9. US Government Publishing Office. Procedures for determining the bioavailability or bioequivalence of drug products. Available from: http://www.ecfr.gov/cgi-bin/text-idx?SID=2c4f1e03168ddbafbe6af7 $3 \mathrm{~b} 46 \mathrm{e} 6 \mathrm{a} 567 \& \mathrm{mc}=$ true $\&$ node $=\mathrm{se} 21.5 .320 \_122 \& \mathrm{rgn}=\operatorname{div} 8$. Accessed June 8, 2017.

10. US Food and Drug Administration. Inactive ingredient search for approved drug products: frequently asked questions. Available from: http://www.fda.gov/Drugs/InformationOnDrugs/ucm080123.htm. Updated July 14, 2017. Accessed June 8, 2017.

11. Ahsan N, Palmer BF, Wheeler D, Greenlee RG Jr, Toto RD. Intravenous immunoglobulin-induced osmotic nephrosis. Arch Intern Med. 1994;154(17):1985-1987.

12. Bornet F, Haardt MJ, Costagliola D, Blayo A, Slama G. Sucrose or honey at breakfast have no additional acute hyperglycaemic effect over an isoglucidic amount of bread in type 2 diabetic patients. Diabetologia. 1985;28(4):213-217.

13. Chantelau EA, Gosseringer G, Sonnenberg GE, Berger M. Moderate intake of sucrose does not impair metabolic control in pump-treated diabetic out-patients. Diabetologia. 1985;28(4):204-207.
Drug Design, Development and Therapy

\section{Publish your work in this journal}

Drug Design, Development and Therapy is an international, peerreviewed open-access journal that spans the spectrum of drug design and development through to clinical applications. Clinical outcomes, patient safety, and programs for the development and effective, safe, and sustained use of medicines are the features of the journal, which

\section{Dovepress}

has also been accepted for indexing on PubMed Central. The manuscript management system is completely online and includes a very quick and fair peer-review system, which is all easy to use. Visit http://www.dovepress.com/testimonials.php to read real quotes from published authors.

Submit your manuscript here: http://www.dovepress.com/drug-design-development-and-therapy-journal 